\title{
Correlative Microscopy of Biological Cells and Tissues by Scanning X-ray Diffraction, Holography, Tomography and Super-Resolution Optical Microscopy
}

\author{
M.Bernhardt ${ }^{1}$, J.-D. Nicolas ${ }^{1}$, M. Osterhoff ${ }^{1}$, H. Mittelstädt ${ }^{2}$, M. Reuss ${ }^{2}$, B. Harke², A. Wittmeier ${ }^{1}$, \\ M. Sprung ${ }^{3}$, S. Köster ${ }^{1}$ and T. Salditt ${ }^{*}$
}

1. Institut für Röntgenphysik, Universität Göttingen, Göttingen, Germany

2. Abberior Instruments, Göttingen, Germany ${ }^{3 .}$ DESY, Hamburg, Germany

* Corresponding author, tsaldit@gwdg.de

We present a correlative microscopy approach for biological cells and tissues, which combines holographic x-ray imaging, x-ray scanning diffraction, and super-resolution optical fluorescence microscopy, in particular stimulated emission depletion (STED)-microscopy [1]. All three imaging modalities have been integrated into the same dedicated synchrotron nano-focus endstation GINIX at the P10 beamline of the PETRAIII storage ring (DESY, Hamburg) [2], as sketched in Fig.1. This allows us to image both labeled and unlabeled biomolecular assemblies in the cell in a quasi-simultaneous scheme. We exploit the complementary contrast mechanisms of x-ray microscopy and optical fluorescence: While scanning x-ray diffraction and x-ray holographic imaging provide structural information on the native electron-density distribution within the cell, fluorescent markers visualize specific molecular components.

We demonstrate this approach by mapping the fluorescently labeled actin cytoskeleton in heart tissue cells. The optical micrographs of the labeled network are then registered with the projected electron density, which has been reconstructed from x-ray holograms by holographic phase retrieval. Holograms were recorded with x-ray waveguide optics, placed in the focal plane of the KB nano-focus [2]. In addition, cells were scanned in the focal plane, with diffraction patterns recorded in each scan point, covering the q-range of small-angle x-ray scattering (SAXS). These patterns can be interpreted in terms of biomolecular shape and spatial correlations of all scattering constituents contributing to the signal. We find that the principal directions of the anisotropic diffraction patterns coincide to a certain extent with the actin fiber directions. Further, actin filaments bundles are also recognizable in the phase map reconstructed from holographic recordings. We currently investigate how the in situ STED recordings can help us to formulate suitable models for the diffraction data, based on co-localisation constraints. They also allow us to monitor radiation damage during the experiment, as reported in more detail elsewhere.

The additional information by super-resolution optical microscopy can be used to constrain models and parameters in x-ray data analysis. Since the entire biomolecular ensemble in the illuminated spot contributes to the diffraction and/or the x-ray phase shift, it is often difficult or impossible to formulate models for the diffraction without additional information. For this reason, most of our previous studies of biological cells by scanning x-ray diffraction were analyzed in terms in a fully automized, but model-free approach. To this end, different parameters are extracted from the diffraction patterns (DP), such as total diffraction intensity (darkfield), differential phase contrast (DPC), second moments of the scattering distribution, power-law exponents, or anisotropy parameters based on principal component analysis (PCA) [3]. By inspection of the DP and the real-space maps, it seems plausible to attribute diffraction signals in some locations to the presence of filamentous proteins of the cytoskeleton. However, such conclusions need confirmation by optical fluorescence microscopy, at highest possible resolution. With this information at hand, the local diffraction patterns can be interpreted and analysed, providing in the end more information than the optical image, for example on structural parameters of filament bundles or 
on DNA compactification. To this end, we refer to recent work on protein filament bundles [3,4], lattice spacings of the acto-myosin lattice in muscle [5,6], or on the electron density of specific organelles [7], where optical microscopy was essential, even if not available in the current quality and resolution.

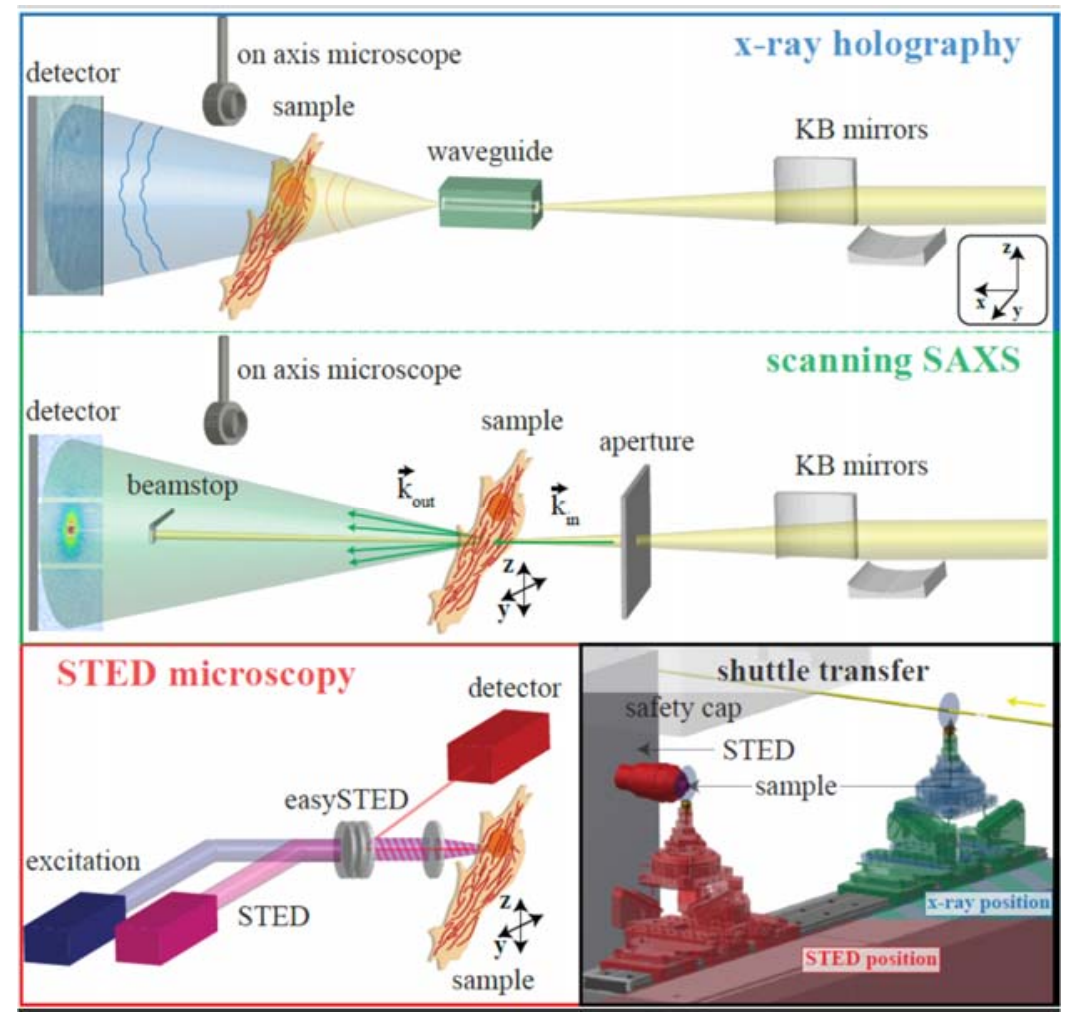

Figure. 1. Schematic of all three imaging modalities: x-ray holography (blue box), scanning SAXS (green box) and STED-microscopy (red box). The STED optical axis is aligned antiparallel to the $\mathrm{x}$-ray beam. Switching between STED-microscopy and the two x-ray modalities requires a lateral shuttle transfer of the sample tower, by a hexapod-nanopositioning system. First, a STED-image is recorded, followed by s low dose x-ray holography recording and finally a high dose scanning SAXS scan (with the sample in the KB focus). For scanning SAXS and STED, the sample is scanned, while holography and tomography are recorded in fullfield mode, with the object in a defocus position with respect to the $\mathrm{x}$-ray waveguide positioned in the $\mathrm{KB}$ foal plane.

The concept of the correlative microscopy approach is shown in Fig.1, and reported in full detail elsewhere. The STED microscope is aligned anti-parallel to the x-ray beam, in close proximity to the xray recording position of the sample. The microscope consists of an excitation and a depletion (STED-) laser with wavelengths of 640 and $775 \mathrm{~nm}$ respectively. The beams are focused by a NA=0.95/40x air objective (UPLSAPO,Olympus) onto the sample. The STED laser is shaped by a set of waveplates exploiting polarization properties of the beam (so-called 'easySTED'-principle), resulting in a doughnutlike intensity profile in the focal plane. The STED-pulse impinges on the sample with a controlled time delay on the order of hundreds of picoseconds with respect to the excitation pulse. It depletes the fluorescent signal by stimulated emission in the entire annulus around the central zero, leaving only the fluorescence signal from the central zero is to be detected by the photon-counting avalanche photodiode (APD). This principle results in an effective probing spot significantly below the diffraction limit. For the current implementation, the beam has to traverse a small air gap of $180 \mu \mathrm{m}$ in order to facilitate the shuttle between the STED and x-ray position. However, regardless of the absolute NA and resolution, the relative resolution advantage over confocal microscopy is maintained for a given NA, and significantly more features of the actin cytoskeleton can be visualized by STED when compared to a confocal scan, see Fig.2. As in other STED microscopes, confocal recordings are used as controls alongside with the STED recording. In addition, bright field and epifluorescence images can be acquired in a full-field mode of the microscope for inspection of larger areas during initial alignment. The STED add-on to the GINIX endstation enables a quick motorized switch between all three imaging modes, see also Fig.1.The data shown 
here was obtained on neonatal rat cardiac tissue cells with labelled actin and prepared by plunge freezing followed by freeze-drying to keep the experiment simple for a first demonstration. The KB focal spot size was in the range of 300nm, and the photon energy was $13.8 \mathrm{keV}$.

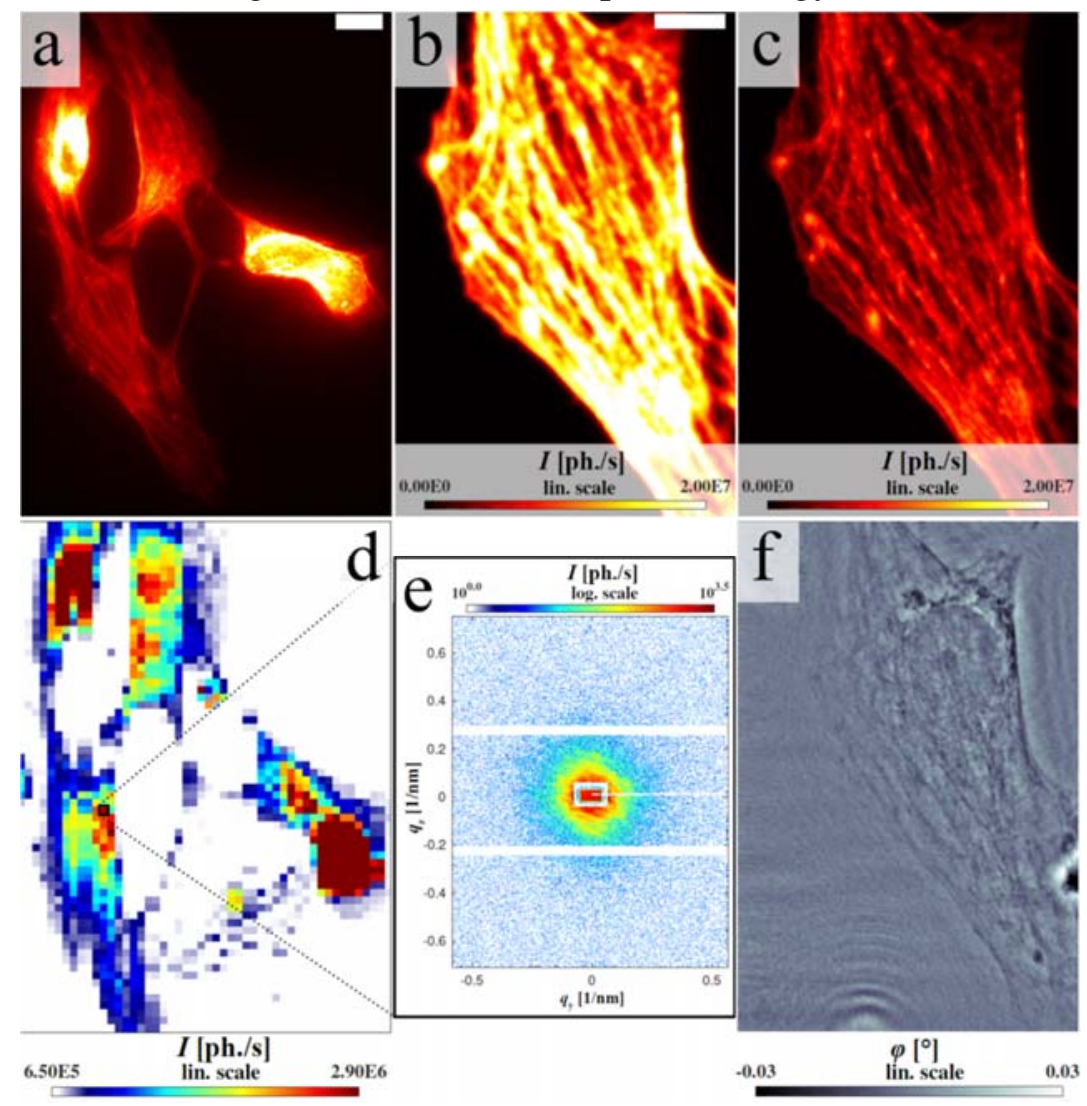

Figure. 1. (a) Epifluorescence micrograph (of neonatal rat cardiac tissue cells with labeled actin cytoskeleton (Phalloidin-Atto633, AD 633-8X, Atto-tec). Cells adhering on glass were fixated, labeled, plungefrozen and freeze-dried, to facilitate this first proof of concept experiment. Scale bar: $10 \mu \mathrm{m}$. (b) Confocal-, and (c) STED-micrograph, of the cell in the lower left half of (a). Scale bar of (b): $5 \mu \mathrm{m}$. (d) X-ray dark field of the scan area as obtained by integration of scattered photons of each local SAXS pattern. (e) Single 2D diffraction pattern of the local spot marked in (d). Analysis of the SAXS pattern will be reported elsewhere (f) Preliminary phase map (available during the experiment) by single distance CTFbased phase phase retrieval of x-ray holograms.

In future, STED microscopy could also be combined with live cell holographic x-ray recordings [8], as well as with holo-tomography of fixated cells [9], provided suitable holders or chambers.

\section{References:}

[1] S. W. Hell and J. Wichmann. Opt Lett, 19, 780 (1994).

[2] T. Salditt, M. Osterhoff, M. Krenkel, R. N. Wilke, M. Priebe, M. Bartels, S. Kalbfleisch, M. Sprung. J.Synchr.Rad. 22, 867 (2015).

[3] M.Bernhardt, M.Priebe,M.Osterhoff,C.Wollnik,A.Diaz,T.Salditt,F.Rehfeldt. Biophys. J.10, 680 (2016).

[4] B.Weinhausen, O.Saldanha, R.N.Wilke, C.Dammann, M.Priebe, M.Burghammer, M.Sprung, S.Köster. Phys.Rev. Lett. 112, 088102 (2014).

[5] M.Bernhardt, J.D.Nicolas, M.Eckermann, B.Eltzner, F.Rehfeldt, T.Salditt. N. J. of Physics 19, 013012 (2017).

[6] J.D.Nicolas, M.Bernhardt, A.Markus, F.Alves, M.Burghammer, T.Salditt. J.Synchr.Rad. 24, 1163 (2017).

[7] R.N.Wilke, M.Priebe, M.Bartels, K.Giewekemeyer, A.Diaz, P.Karvinen, T.Salditt. Opt. Ex. 20, 19232 (2012).

[8] M.Bartels, M.Krenkel, J.Haber, R.N.Wilke,T.Salditt, Phys.Rev.Lett. 114, 048103 (2015).

[9] M. Krenkel, M. Toepperwien, F. Alves, and T. Salditt. Acta Crystallogr A 73, 282 (2017).

[10]We acknowledge SFB937 /A11 for funding the correlative microscopy of actin cytoskeleton networks, and support through BMBF project 05K16MG2 for the custom-built STED-microscope. We thank Bastian Hartmann, Peter Luley, and Matthias Meister for engineering support. 
https://doi.org/10.1017/S1431927618012734 Published online by Cambridge University Press 